\title{
Pseudoarthrosis and fracture: interaction between severe vitamin D deficiency and primary hyperparathyroidism
}

Ashu Rastogi ${ }^{1}$, MD, DM, Sanjay Kumar $\underline{\text { Bhadada }}^{1}$, MD, DM, Anil Bhansali ${ }^{1}$, MD, DM

\begin{abstract}
A young woman with severe vitamin D deficiency presented with proximal muscle weakness, fragility fracture and pseudoarthrosis. On evaluation, she was found to have hypercalcaemia, a single parathyroid adenoma and an undetectable 25-hydroxyvitamin D level. She received parenteral cholecalciferol and subsequently underwent curative parathyroidectomy. Postoperatively, she had hungry bone syndrome, which she gradually recovered from with calcium and calcitriol replacement. Notably, her calcium levels were in the lower limit of normal range and associated with elevated alkaline phosphatase levels at postoperative Day 14. Follow-up for the next four years showed that the patient had remarkable symptomatic and radiological improvements. In this report, we discuss the pathophysiological interactions between vitamin D deficiency and associated primary hyperparathyroidism.
\end{abstract}

Keywords: pseudoarthrosis, primary hyperparathyroidism, vitamin D deficiency

\section{INTRODUCTION}

Vitamin D deficiency, especially those due to nutritional causes, is still common in developing countries. It is biochemically characterised by hypophosphataemia, normo/hypocalcaemia, raised alkaline phosphatase and secondary hyperparathyroidism. ${ }^{(1-3)}$ Biochemically, vitamin D deficiency is defined as serum 25-hydroxyvitamin D levels $<20 \mathrm{ng} / \mathrm{mL}$, while insufficiency is defined as $20-29 \mathrm{ng} / \mathrm{mL}$. ${ }^{(4)}$ Although symptomatic presentation of primary hyperparathyroidism (PHPT) is uncommon in Western countries, ${ }^{(5)}$ vitamin D deficiency is common among PHPT patients compared to the general population. ${ }^{(6-8)}$ Vitamin D deficiency is associated with a more severe biochemical form of PHPT, which may present with bony features. Nordenström et $\mathrm{a}^{\left({ }^{(9)}\right.}$ reported that low serum levels of 25-hydroxyvitamin D in the 10-year period preceding parathyroidectomy was an independent risk factor for the occurrence of fractures and extensive bony lesions. Fractures and pseudoarthrosis are rare skeletal manifestations of vitamin D deficiency. ${ }^{(1,6)}$ We report a case of severe vitamin D deficiency with symptomatic PHPT, and discuss the pathophysiological interactions between the two entities.

\section{CASE REPORT}

A 30-year-old woman presented with low backache and progressively worsening proximal muscle weakness that had persisted for five years. Six months prior to presentation, she had sustained a fragility fracture of the left ulna. She had no history of any focal neurological deficits, seizures or carpopedal spasms. The patient was from a low socioeconomic stratum
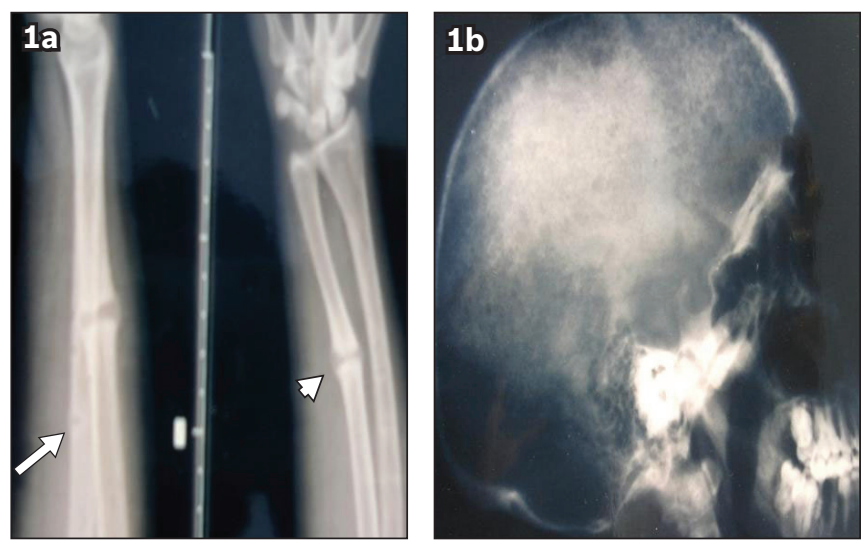

Fig. 1 (a) Radiographs show Looser's zones (arrow) in the forearm bones and pseudoarthrosis (arrowhead) in the shaft of the ulna. (b) Lateral skull radiograph shows the classical 'salt and pepper' appearance caused by hyperparathyroidism.

and had very poor nutritional intake. Her calcium intake and exposure to sunlight was grossly inadequate. In the last five years, she had two pregnancies and a history of prolonged (> 2 years) lactation. There was no suggestion of malabsorption, use of anticonvulsant medications, neck surgery, renal stone disease or psychiatric illness. The patient had been taking calcium and cholecalciferol intermittently. There was no history of renal stone disease, pancreatitis or fragility fractures in her family. She was lean with a weight, height and body mass index of $53 \mathrm{~kg}, 157 \mathrm{~cm}$ and $21.5 \mathrm{~kg} / \mathrm{m}^{2}$, respectively.

Physical examination revealed that she had a deformity of the left ulna and severe proximal muscle weakness of the lower limbs with normal reflexes. Signs of latent tetany (Chvostek's or Trousseau's sign) were absent, and the rest of her

${ }^{1}$ Department of Endocrinology, Post Graduate Institute of Medical Education and Research, Chandigarh, India

Correspondence: Dr Sanjay Kumar Bhadada, Associate Professor, Department of Endocrinology, Post Graduate Institute of Medical Education and Research, Chandigarh 160012, India. bhadadask@rediffmail.com 
Table I. Postoperative biochemical profile of the patient.

\begin{tabular}{|c|c|c|c|c|c|c|c|}
\hline \multirow{2}{*}{$\begin{array}{l}\text { Laboratory } \\
\text { investigation }\end{array}$} & \multicolumn{7}{|c|}{ Postoperative day } \\
\hline & 1 & 2 & 3 & 4 & 7 & 10 & 14 \\
\hline Calcium (mg/dL) & 8.9 & 8.1 & 7.9 & 8.4 & 8.4 & 8.5 & 8.7 \\
\hline $\begin{array}{l}\text { Phosphorus } \\
\text { (mg/dL) }\end{array}$ & 3.3 & 2.3 & 2.3 & 2.7 & 3.0 & 3.0 & 3.4 \\
\hline $\begin{array}{l}\text { Alkaline phosphatase } \\
(U / L)\end{array}$ & 277 & 256 & 550 & 1,022 & 432 & 330 & 387 \\
\hline Albumin* $(\mathrm{g} / \mathrm{dL})$ & 4.1 & 4.1 & 4.3 & 4.0 & 4.1 & 4.1 & 4.2 \\
\hline iPTH (pg/mL) & - & - & 70 & - & - & - & 56 \\
\hline $\begin{array}{l}\text { 25-hydroxyvitamin D } \\
\text { (ng/mL) }\end{array}$ & - & - & - & - & - & - & 10.1 \\
\hline
\end{tabular}

${ }^{\star}$ Normal range 3.5-4.5 g/dL. iPTH: intact parathyroid hormone

systemic examination was unremarkable. Investigation showed mean serum calcium level (corrected for serum albumin) at $11.2 \mathrm{mg} / \mathrm{dL}$ (normal range $[\mathrm{NR}]$ 8.5-10.1 $\mathrm{mg} / \mathrm{dL}$ ), mean serum phosphorus level at $2.4 \mathrm{mg} / \mathrm{dL}$ (NR $2.5-4.5 \mathrm{mg} / \mathrm{dL}$ ), and mean serum alkaline phosphatase at $670.4 \mathrm{U} / \mathrm{L}$ (NR 42-128 U/L). The patient's renal, liver and thyroid function tests were unremarkable. Serum 25-hydroxyvitamin D level was $<4 \mathrm{ng} / \mathrm{mL}(\mathrm{NR} 9.1-41.3 \mathrm{ng} / \mathrm{mL})$ and serum intact parathyroid hormone level (iPTH) was 1,742 pg/mL (NR 7-52 pg/mL). Plain radiographs of the patient's hand and forearm showed Looser's zones (pseudofractures), a true fracture with pseudoarthrosis in the left ulna (Fig. 1a), and subperiosteal resorption of the phalanges. Plain radiograph of the skull (lateral view) showed a classical 'salt and pepper' appearance, which is typical of PHPT (Fig. 1b). Ultrasonography of the patient's neck showed a hypoechoic focus in the right lower pole of the thyroid, suggestive of a parathyroid adenoma. The parathyroid adenoma was subsequently confirmed using thallium-technetium subtraction imaging of the same site.

Surgical exploration of the neck was performed, and a single parathyroid adenoma measuring $2 \mathrm{~cm} \times 1 \mathrm{~cm}$, found in the right lower parathyroid position, was excised. The remaining parathyroid glands were observed to be small and atrophic. The excised parathyroid adenoma weighed 9.6 g. Histopathology of the specimen confirmed chief cell tumour without capsular or vascular invasion. There was no evidence of periadenomatous hyperplasia. On postoperative Day 2, the patient developed latent tetany with decreased serum calcium and phosphate levels, as well as a trend of increasing alkaline phosphatase, which was suggestive of hungry bone syndrome (HBS). There was a decrease in her iPTH level $(70 \mathrm{pg} / \mathrm{mL})$ and an increase in 25-hydroxyvitamin D level $(10.1 \mathrm{ng} / \mathrm{mL}$ ) (Table I). The patient's symptoms of HBS improved by postoperative Day 7 , after supplementation with calcitriol $(0.5 \mathrm{mcg} /$ day $)$ and calcium carbonate (2 g/day) in divided doses. Her calcium level was in the low-normal range, while her alkaline phosphatase level remained high up to postoperative Day 14. The patient recovered well with healing of the Looser's zones. She experienced no fractures over the past four years of follow-up and is ambulatory.
Table II. Differences between Looser's zones and true fractures.

\begin{tabular}{lll}
\hline Characteristic & Looser's zones & True fractures \\
\hline Direction & Perpendicular & Can be oblique \\
Lucent band & Present & Usually absent \\
Margins & Parallel & Can diverge \\
Adjacent bone & Normal & Can be abnormal \\
Number & Multiple & Usually single \\
Sclerosis & Absent & Present \\
Symmetry & Present & Absent \\
Visible callus & Absent & Present \\
Healing & Slow or absent & Rapid \\
\hline
\end{tabular}

\section{DISCUSSION}

Although severe nutritional vitamin D deficiency with PHPT is still common in developing countries, it is rarely associated with pseudoarthrosis. Vitamin D deficiency is characterised by hypophosphataemia, hypo- or normocalcaemia, raised serum alkaline phosphatase levels and serum 25-hydroxyvitamin D levels $<20 \mathrm{ng} / \mathrm{mL}$. Nutritional factors such as inadequate vitamin D intake and lack of sufficient exposure to sunlight are the main causes of vitamin D deficiency in developing countries like India. (1-3) Patients usually present with bony pains, proximal muscle weakness, and in children, bony deformities. Patients rarely present with fractures. Osteomalacia is also commonly seen in patients with vitamin D deficiency. Radiologically, the presence of Looser's zones is one of the characteristic abnormalities seen in osteomalacia, in addition to osteopenia, widening of the epiphysis, and 'cupping and/or fraying' at the metaphyseal end of the bones. ${ }^{(1)}$ Looser's zones are usually seen along the course of the traversing artery. They have smooth edges and present across the width of the bone. Importantly, Looser's zones are bilaterally symmetrical and can be differentiated from true fractures in most cases (Table II).

The manifestations of PHPT are varied and seldom symptomatic in Western populations nowadays. ${ }^{(5)}$ Bone involvement is not frequently seen in Western studies, as PHPT is diagnosed early by multiphasic screening in the West. One of the main postulates for the florid bone manifestations of PHPT in developing countries is the high prevalence of vitamin D deficiency, with pseudoarthrosis being one of the rarer findings in this form of metabolic bone disease. ${ }^{(1-3)}$ Pseudoarthrosis may be a nonspecific complication resulting from poor healing of fractured bones, and is exacerbated by inadequate immobilisation, as seen in our patient.

Although the coexistence of vitamin D deficiency and symptomatic PHPT is uncommon, it is well described and occurs more frequently in developing countries. ${ }^{(1,3,6-8)}$ In vitamin D deficiency, secondary hyperparathyroidism usually occurs due to the lack of negative feedback of both active vitamin $\mathrm{D}$ and calcium on the parathyroid cells. This maintains the serum calcium level in the normal range (unless there is severe vitamin D deficiency when hypocalcaemia occurs) and causes hypophosphataemia (due to the phosphaturic effect of 
parathyroid hormone). However, if hypercalcaemia occurs in the setting of vitamin D deficiency, as was seen in our case, two possibilities should be considered: (a) secondary hyperparathyroidism had evolved into an autonomous nodule (i.e. tertiary hyperparathyroidism), ${ }^{(10)}$ or (b) PHPT.

In the setting of severe vitamin D deficiency, only $50 \%$ of PHPT cases manifest signs of hypercalcaemia, which is one of the important causes of normocalcaemic PHPT.(1) A possible reason for the hypercalcaemia observed in our patient with severe vitamin D deficiency is increased osseous tissue response due to overwhelming PTH levels, and intermittent calcium and vitamin D therapy. As primary and tertiary hyperparathyroidism cannot be differentiated clinically, histopathology is required for confirmation. In tertiary hyperparathyroidism, there is hyperplasia of the remaining parathyroid glands in the presence of a single adenoma. In our patient, histopathology of the excised gland showed chief cell adenoma with no evidence of periadenomatous hyperplasia and the remaining parathyroid glands were small and atrophic, thus confirming the diagnosis of PHPT.

It has been postulated that chronic vitamin D deficiency may result in calcium-sensing receptor abnormalities, leading to excessive production of PTH, or vice versa. The causes of low circulating levels of serum 25-hydroxyvitamin D levels in patients with PHPT are not fully understood. PHPT may accentuate vitamin D deficiency through an accelerated conversion of 25-hydroxyvitamin D to 1,25-dihydroxyvitamin D, which depletes vitamin D stores. ${ }^{(11)}$ In addition, increased adiposity described in patients with PHPT might be a contributing factor, as vitamin D may be sequestered in adipose tissue. $^{(12)}$

The Third International Workshop on Asymptomatic PHPT recommended that serum concentrations of 25-hydroxyvitamin D be measured in all patients with suspected PHPT, and that vitamin $\mathrm{D}$ supplementation be initiated for patients with 25-hydroxyvitamin D levels below $20 \mathrm{ng} / \mathrm{mL}$ before any medical or surgical decisions are made.(13) In view of our patient's severe vitamin D deficiency, she was given cholecalciferol supplements preoperatively despite having PHPT. A study by Mikhail ${ }^{(14)}$ showed that vitamin D supplementation in cases of mild PHPT was safe; however, most of the patients in this study had asymptomatic PHPT with serum calcium levels that were $<12 \mathrm{mg} / \mathrm{dL}$. While vitamin $\mathrm{D}$ therapy has been associated with a mild decrease in serum PTH levels (mean reduction range 3\%-25\%), vitamin D replacement prior to parathyroidectomy had no significant effect on cure rates, frequency of recurrent disease, or occurrence of eucalcemic PTH elevation after parathyroidectomy. ${ }^{(15)}$ In our study, we could not repeat the measurement of the patient's serum 25-hydroxyvitamin D levels preoperatively after supplementation, as the patient was subjected to surgery on the third day of cholecalciferol supplementation.

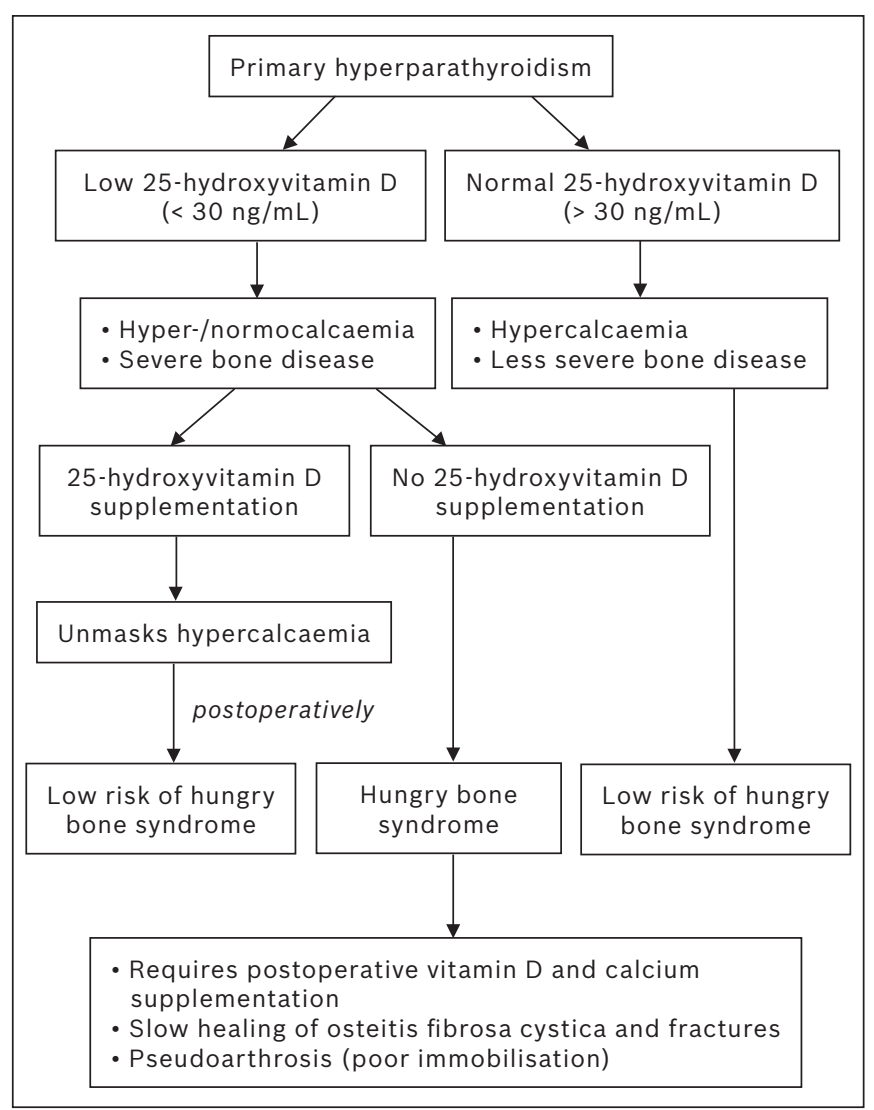

Fig. 2 Flowchart shows the interaction between vitamin D and primary hyperparathyroidism.

Postoperatively, the patient developed signs of latent tetany and had hypocalcaemia with raised alkaline phosphatase. These are characteristic features of HBS, which commonly occur if there is extensive bone involvement. The patient was managed expectantly with preoperative administration of calcium carbonate and vitamin D, especially 1,25-dihydroxyvitamin D, as it has an easily titratable short half-life. In severe vitamin D deficiency, HBS may last for a longer duration and may require prolonged calcium supplementation, as was observed in our patient. Fig. 2 summarises the interaction between vitamin D and PHPT.

In conclusion, vitamin D deficiency may alter the classical presentation of PHPT. Vitamin D-deficient individuals may have greater severity of PHPT, as well as a higher risk of severe postoperative HBS.

\section{REFERENCES}

1. Harinarayan CV, Gupta N, Kochupillai N. Vitamin D status in primary hyperparathyroidism in India. Clin Endocrinol (Oxf) 1995; 43:351-8.

2. Shah VN, Bhadada SK, Bhansali A, et al. Influence of age and gender on presentation of symptomatic primary hyperparathyroidism. J Postgrad Med 2012; 58:107-11.

3. Fouda MA. Primary hyperparathyroidism and vitamin D deficiency: A combination still encountered in Asian countries. Ann Saudi Med 1999; 19:455-8.

4. Holick MF, Binkley NC, Bischoff-Ferrari HA, et al. Evaluation, treatment, and prevention of vitamin D deficiency: an Endocrine Society clinical practice guideline. J Clin Endocrinol Metab 2011; 96:1911-30.

5. Wermers RA, Khosla S, Atkinson EJ, et al. The rise and fall of primary hyperparathyroidism: a population-based study in Rochester, Minnesota, 1965-1992. Ann Intern Med 1997; 126:433-40. 
6. Moosgaard B, Vestergaard P, Heickendorff L, et al. Vitamin D status, seasonal variations, parathyroid adenoma weight and bone mineral density in primary hyperparathyroidism. Clin Endocrinol (Oxf) 2005; 63:506-13.

7. Boudou P, Ibrahim F, Cormier C, Sarfati E, Souberbielle JC. A very high incidence of low 25 hydroxy-vitamin D serum concentration in a French population of patients with primary hyperparathyroidism. J Endocrinol Invest 2006; 29:511-5.

8. Kandil E, Tufaro AP, Carson KA, et al. Correlation of plasma 25hydroxyvitamin $\mathrm{D}$ levels with severity of primary hyperparathyroidism and likelihood of parathyroid adenoma localization on sistamibi scan. Arch Otolaryngol Head Neck Surg 2008; 134:1071-5.

9. Nordenström E, Westerdahl J, Lindergård B, Lindblom P, Bergenfelz A. Multifactorial risk for bone fractures in primary hyperparathyroidism. World J Surg 2002; 26:1463-7.

10. Ludwig GD, Kyle GC, de Blanco M. "Tertiary" hyperparathyroidism induced by osteomalacia resulting from phosphorus depletion. Am J
Med 1967; 43:136-40.

11. Davies M, Heys SE, Selby PL, Berry JL, Mawer EB. Increased catabolism of 25-hydroxyvitamin $\mathrm{D}$ in patients with partial gastrectomy and elevated 1,25-dihydroxyvitamin D levels. Implications for metabolic bone disease. J Clin Endocrinol Metab 1997; 82:209-12.

12. Bolland MJ, Grey AB, Gamble GD, Reid IR. Association between primary hyperparathyroidism and increased body weight: a meta-analysis. J Clin Endocrinol Metab 2005; 90:1525-30.

13. Eastell R, Arnold A, Brandi ML, et al. Diagnosis of asymptomatic primary hyperparathyroidism: proceedings of the third international workshop. J Clin Endocrinol Metab 2009; 94:340-50.

14. Mikhail N. Clinical significance of vitamin D deficiency in primary hyperparathyroidism and safety of Vitamin D therapy. Southern Med J 2011; 104:29-33.

15. Grubbs EG, Rafeeq S, Jimenez C, et al. Preoperative vitamin D replacement therapy in primary hyperparathyroidism: safe and beneficial? Surgery 2008; 144:852-8. 